\title{
Boundedness and Global Exponential Stability for Delayed Differential Equations with Applications
}

\author{
Teresa Faria $^{a, \star *}$ and José J. Oliveira ${ }^{b, \dagger}$ \\ ${ }^{a}$ Departamento de Matemática and CMAF, Faculdade de Ciências, \\ Universidade de Lisboa, Campo Grande, 1749-016 Lisboa, Portugal \\ e-mail: tfaria@ptmat.fc.ul.pt \\ ${ }^{b}$ Departamento de Matemática, Escola de Ciências, \\ Universidade do Minho, Campus de Gualtar, 4710-057 Braga, Portugal \\ e-mail: jjoliveira@math.uminho.pt
}

\begin{abstract}
The boundedness of solutions for a class of $n$-dimensional differential equations with distributed delays is established, by assuming the existence of instantaneous negative feedbacks which dominate the delay effect. As an important by-product, some criteria for global exponential stability of equilibria are obtained. The results are illustrated with applications to delayed neural networks and population dynamics models.

Keywords: global exponential stability, global asymptotic stability, delay, neural network, Cohen-Grossberg neural network, delayed population model.

2000 AMS Subject Classification: 34K20, 34K25, 34K60, 92B20, 92D25.

\section{Introduction}

Let $C_{n}:=C\left([-\tau, 0] ; \mathbb{R}^{n}\right)$ be the space of continuous functions from $[-\tau, 0]$ to $\mathbb{R}^{n}, \tau>0$, equipped with the supremum norm $\|\varphi\|=\max _{-\tau \leq \theta \leq 0}|\varphi(\theta)|$, for any fixed norm $|\cdot|$ in $\mathbb{R}^{n}$. As usual, $x_{t} \in C_{n}$ is defined by $x_{t}(\theta)=x(t+\theta),-\tau \leq \theta \leq 0$.

In this paper we are particularly interested in situations modelled by systems of functional differential equations (FDEs) of the form

$$
\dot{x}_{i}(t)=-\rho_{i}\left(t, x_{t}\right)\left[b_{i}\left(x_{i}(t)\right)+f_{i}\left(t, x_{t}\right)\right], \quad t \geq 0, \quad i=1, \ldots, n,
$$

* Corresponding author. Fax:+351 21795 4288; Tel: +351 217904894.

* Work partially supported by FEDER and Fundação para a Ciência e Tecnologia (FCT)-Plurianual 2007, under CMAF.

$\dagger$ Work partially supported by FCT, under grant SFRH/BD/29563/2006, CMAT, and programme POCI 2010.
\end{abstract}


where $b_{i}: \mathbb{R} \rightarrow \mathbb{R}, \rho_{i}, f_{i}:[0, \infty) \times C_{n} \rightarrow \mathbb{R}$ are continuous, with $\rho_{i}(t, \varphi)$ positive, $i=1, \ldots, n$. We further assume that the negative feedback condition $b_{i}(x) x>0$ for $x \neq 0, i=1, \ldots, n$, is satisfied.

System (1.1) is sufficiently general to include as particular classes some well-known models used in population dynamics and neural networks, such as delayed Hopfield and Cohn-Grossberg models.

If (1.1) corresponds to an $n$-species population model, due to its biological interpretation only positive solutions are meaningful and therefore admissible. In a general setting, for an FDE in $C_{n}$,

$$
\dot{x}(t)=f\left(t, x_{t}\right), \quad t \geq t_{0},
$$

with $f: D \subset \mathbb{R} \times C_{n} \rightarrow \mathbb{R}^{n}$ continuous, we often fix a set $S \subset C_{n}$, with $\left[t_{0}, \infty\right) \times S \subset D$, as the set of admissible initial conditions. Naturally, a solution $x(t)$ with an admissible initial condition $x_{t_{0}}=\varphi \in S$ is said to be admissible if $x_{t} \in S$ for $t>t_{0}$ whenever $x_{t}$ is defined. Under the assumption of uniqueness of solutions, the solution of (1.2) with initial condition $x_{t_{0}}=\varphi, \varphi \in S$, is denoted by $x\left(t, t_{0}, \varphi\right)$.

Assuming that (1.1) has an admissible equilibrium $x^{*}$, our main purpose is to establish sufficient conditions for its global exponential or asymptotic stabilities, whose definitions we recall here.

Definition 1.1. If $x^{*} \in S$ is an equilibrium of (1.1), $x^{*}$ is said to be globally exponentially stable (in the set $S$ of admissible solutions) if there are positive constants $\varepsilon, M$ such that

$$
\left|x(t, 0, \varphi)-x^{*}\right| \leq M e^{-\varepsilon t}\left\|\varphi-x^{*}\right\|, \quad \text { for all } t \geq 0, \varphi \in S
$$

and $x^{*}$ is said to be globally asymptotically stable if it is stable and $x(t) \rightarrow x^{*}$ as $t \rightarrow \infty$, for all admissible solutions $x(t)$.

The present paper is inspired by a result of the authors in [7], which establishes a criterion for boundedness of solutions to (1.2). As shown here, such criterion can also be used to derive the global exponential stability of equilibria, and has applications to numerous relevant models, either recovering or improving results in recent literature normally obtained by construction of Lyapunov functionals. Although a very effective technique for proving boundedness of solutions and global attractivity of equilibria, the construction of a Lyapunov functional is in general a very difficult task, and involves long computations. Moreover, a new Lyapunov functional for each model under consideration is often required. On the contrary, our approach does not require the use of Lyapunov functional methods, and establishes simple criteria applicable to a quite general framework.

We now set some notation. For $c \in \mathbb{R}^{n}$, we use both $c$ and $\hat{c}$ to denote the constant function $\varphi(\theta)=c$ in $C_{n}$. A vector $d=\left(d_{1}, \ldots, d_{n}\right) \in \mathbb{R}^{n}$ is said to be positive if $d_{i}>0$ for $i=1, \ldots, n$, and we write $d>0$. 
Definition 1.2. For $d=\left(d_{1}, \ldots, d_{n}\right)>0$, define the inner product $<x, y>_{d}=\sum_{i=1}^{n} d_{i} x_{i} y_{i}$ for $x=\left(x_{1}, \ldots, x_{n}\right), y=\left(y_{1}, \ldots, y_{n}\right) \in \mathbb{R}^{n}$, with associated norm $|x|_{2, d}=\left(\sum_{i=1}^{n} d_{i} x_{i}^{2}\right)^{1 / 2}$; define also the norm $|x|_{\infty, d}=\max _{i=1}^{n} d_{i}\left|x_{i}\right|$.

For $\mathbb{R}^{n}$ with the norms $|\cdot|_{2, d}$, respectively $|\cdot|_{\infty, d}$ (where $d \in \mathbb{R}^{n}, d>0$ ), we denote the corresponding norms in $C_{n}$ by $\|\cdot\|_{2, d}$, respectively $\|\cdot\|_{\infty, d}:\|\varphi\|_{2, d}=\max _{-\tau \leq \theta \leq 0}|\varphi(\theta)|_{2, d}$ and $\|\varphi\|_{\infty, d}=\max _{-\tau \leq \theta \leq 0}|\varphi(\theta)|_{\infty, d}$. Clearly, all these norms are equivalent norms in $C_{n}$. If $d_{i}=$ $1,1 \leq i \leq n$, we write simply $|\cdot|_{2}$ and $|\cdot|_{\infty}$ for the usual Euclidean and maximum norms in $\mathbb{R}^{n}$, respectively, and $\|\cdot\|_{2}$ and $\|\cdot\|_{\infty}$ for the corresponding norms in $C_{n}$.

If $D=\left[d_{i j}\right]$ is a square matrix with non-positive off-diagonal entries, i.e., $d_{i j} \leq 0$ for all $i \neq j$, we say that $D$ is a non-singular $M$-matrix if all the eigenvalues of $D$ have a positive real part. The latter is also equivalent to saying that there is a positive vector $c$ such that $N c>0$ [8].

We should mention that in [7] the space $\mathbb{R}^{n}$ is equipped with a norm $|\cdot|_{\infty, d}$, whereas here results are obtained for norms of both types $|\cdot|_{2, d}$ and $|\cdot|_{\infty, d}$. For related results for $\mathbb{R}^{n}$ with $|\cdot|_{2}$, see $[15,20-22]$. This raises the question of whether it is possible to obtain similar results for other norms (cf. $[2,4,5,10,12,20]$ ), a problem to be addressed in future work.

We give a summary of the remainder of this paper. In Section 2, for each one of the norms $|\cdot|_{2, d}$ and $|\cdot|_{\infty, d}$, we present a criterion for boundedness of solutions to (1.2), which is then applied to the particular case of FDEs in the form (1.1). As shown in Section 3, as a by-product of such results we deduce sufficient conditions for the global exponential stability of an (admissible) equilibrium point $x^{*}$ of (1.1), if it exists. In Section 4, we illustrate the results with well-known $n$-dimensional neural network models; although with less success, we also apply our ideas to FDEs from population dynamics. Finally, the last section presents some examples, namely a model where the advantage of working with both norms $|\cdot|_{2, d}$ and $|\cdot|_{\infty, d}$ is exploited.

\section{Boundedness of solutions}

Consider an FDE in $\mathbb{R}^{n}$

$$
\dot{y}(t)=f\left(t, y_{t}\right), \quad t \geq t_{0},
$$

where $f: D \subset \mathbb{R} \times C_{n} \rightarrow \mathbb{R}^{n}$ is continuous. For the sake of simplicity of notation, uniqueness of solutions to (2.1) will be assumed. Fix a set $S \subset C_{n}$, with $\left[t_{0}, \infty\right) \times S \subset D$, as the set of admissible initial conditions for (2.1).

Consider first a norm $|\cdot|_{\infty, d}$ in $\mathbb{R}^{n}, d>0$. Next result was established in [7, Lemma 3.2].

Proposition 2.1. [7] Let $f:\left[t_{0}, \infty\right) \times S \rightarrow \mathbb{R}^{n}$ be continuous, $f=\left(f_{1}, \ldots, f_{n}\right)$. For some $d=\left(d_{1}, \ldots, d_{n}\right)>0$, assume that $f$ satisfies

$(H)_{\infty}$ for $t \geq t_{0}$ and $\varphi \in S$, if $|\varphi(\theta)|_{\infty, d}<|\varphi(0)|_{\infty, d}$ for $\theta \in[-\tau, 0)$, then $\varphi_{i}(0) f_{i}(t, \varphi)<0$ for some $i \in\{1, \ldots, n\}$ such that $|\varphi(0)|_{\infty, d}=d_{i}\left|\varphi_{i}(0)\right|$. 
Then, all admissible solutions of (2.1) are defined and bounded for $t \geq t_{0}$. Moreover, if $y(t)=$ $y\left(t, t_{0}, \varphi\right)(\varphi \in S)$ is an admissible solution of (2.1), then $|y(t)|_{\infty, d} \leq\|\varphi\|_{\infty, d}$ for $t \geq t_{0}$.

The version of Proposition 2.1 with a norm $|\cdot|_{2, d}$ in $\mathbb{R}^{n}$ is as follows:

Proposition 2.2. Suppose that $f:\left[t_{0}, \infty\right) \times S \rightarrow \mathbb{R}^{n}$ is continuous. For some $d=\left(d_{1}, \ldots, d_{n}\right)>$ 0 , assume that $f$ satisfies

$(H)_{2}$ for $t \geq t_{0}$ and $\varphi \in S$, if $|\varphi(\theta)|_{2, d}<|\varphi(0)|_{2, d}$ for $\theta \in[-\tau, 0)$, then $<\varphi(0), f(t, \varphi)>_{d}<0$. Then, all admissible solutions of (2.1) are defined and bounded for $t \geq t_{0}$. Moreover, if $y(t)=$ $y\left(t, t_{0}, \varphi\right)(\varphi \in S)$ is an admissible solution of (2.1), then $|y(t)|_{2, d} \leq\|\varphi\|_{2, d}$ for $t \geq t_{0}$.

Proof. Let $y(t)$ be an admissible solution of $(2.1)$ on $\left[t_{0}-\tau, a\right)$ for some $a>t_{0}$, with $|y(t)|_{2, d} \leq$ $K$ for $t \in\left[t_{0}-\tau, t_{0}\right]$. Suppose that there is $t_{1}>t_{0}$ such that $\left|y\left(t_{1}\right)\right|_{2, d}>K$, and define

$$
T=\min \left\{t \in\left[t_{0}, t_{1}\right]: \max _{s \in\left[t_{0}, t_{1}\right]}|y(s)|_{2, d}=|y(t)|_{2, d}\right\} .
$$

We have $|y(T)|_{2, d}>K$ and $|y(t)|_{2, d}<|y(T)|_{2, d} \quad$ for $\quad t \in\left[t_{0}, T\right)$. Since $\left|y_{T}(\theta)\right|_{2, d}=|y(T+\theta)|_{2, d}<$ $|y(T)|_{2, d}$ for $\theta \in[-\tau, 0)$, by $(\mathrm{H})_{2}$ we have $<y(T), f\left(t, y_{T}\right)>_{d}<0$ for all $t \geq t_{0}$. On the other hand, the definition of $T$ implies that the derivative of the map $t \mapsto|y(t)|_{2, d}^{2}$ at $t=T$ is non-negative. Hence, $<y(T), f\left(T, y_{T}\right)>_{d} \geq 0$, a contradiction. This proves that $y(t)$ is extensible to $\left[t_{0}-\tau, \infty\right)$, with $|y(t)|_{2, d} \leq K$ for all $t>t_{0}$.

Remark 2.1. In particular, we note that Propositions 2.1 and 2.2 are valid if conditions $(\mathrm{H})_{\infty}$ and $(\mathrm{H})_{2}$ are replaced, respectively, by

(h) $)_{\infty}$ for $t \geq t_{0}$ and $\varphi \in S$, if $\|\varphi\|_{\infty, d}=|\varphi(0)|_{\infty, d}>0$, then $\varphi_{i}(0) f_{i}(t, \varphi)<0$, for all $i \in\{1, \ldots, n\}$ such that $|\varphi(0)|_{\infty, d}=d_{i}\left|\varphi_{i}(0)\right|$,

and

$(\mathrm{h})_{2}$ for $t \geq t_{0}$ and $\varphi \in S$, if $\|\varphi\|_{2, d}=|\varphi(0)|_{2, d}>0$, then $<\varphi(0), f(t, \varphi)>_{d}<0$.

We are specially motivated by situations modelled by systems of FDEs of the form

$$
\dot{x}_{i}(t)=-\rho_{i}\left(t, x_{t}\right)\left[b_{i}\left(x_{i}(t)\right)+f_{i}\left(t, x_{t}\right)\right], \quad t \geq 0, \quad i=1, \ldots, n,
$$

where $\rho_{i}:[0, \infty) \times S \rightarrow(0, \infty), b_{i}: \mathbb{R} \rightarrow \mathbb{R}, f_{i}:[0, \infty) \times S \rightarrow \mathbb{R}$ are continuous, for some set $S \subset C_{n}$ of admissible initial conditions.

Proposition 2.3. For (2.2), assume the following assumptions:

(A1) there is an equilibrium point $x^{*} \in S$ of (2.2);

(A2) there are constants $\beta_{i}>0$ such that $\frac{b_{i}(u)-b_{i}(v)}{u-v} \geq \beta_{i}$ for all $u, v \in \mathbb{R}, u \neq v$ and $i=1, \ldots, n$; 
$(A 3)_{\infty}$ there is a vector $d=\left(d_{1}, \ldots, d_{n}\right)>0$ such that $\left|f_{i}(t, \varphi)-f_{i}(t, \psi)\right| \leq l_{i}\|\varphi-\psi\|_{\infty, d}$, for all $t \geq 0, \varphi, \psi \in S$, where the Lipschitz constants $l_{i}$ satisfy $d_{i} l_{i}<\beta_{i}, i=1, \ldots, n$.

Then, all solutions $x(t)=x\left(t, t_{0}, \varphi\right)$ (with $t_{0} \geq 0, \varphi \in S$ ) of (2.2) are defined and bounded on $\left[t_{0}, \infty\right)$ and satisfy $\left|x(t)-x^{*}\right|_{\infty, d} \leq\left\|\varphi-x^{*}\right\|_{\infty, d}$ for $t \geq t_{0}$.

Proof. Let $x^{*}=\left(x_{i}^{*}, \ldots, x_{n}^{*}\right) \in S$ be such that $b_{i}\left(x_{i}^{*}\right)+f_{i}\left(t, x^{*}\right)=0, t \geq 0, i=1, \ldots, n$. By the translation $\bar{x}(t)=x(t)-x^{*},(2.2)$ becomes

$$
\dot{\bar{x}}_{i}(t)=-\rho_{i}\left(t, x^{*}+\bar{x}_{t}\right)\left[b_{i}\left(x_{i}^{*}+\bar{x}_{i}(t)\right)+f_{i}\left(t, x^{*}+\bar{x}_{t}\right)\right], \quad i=1, \ldots, n .
$$

Clearly, (2.3) also has the form (2.2), for which (A1), (A2) and (A3) $)_{\infty}$ hold with $S-x^{*}$ as the set of initial conditions. Hence, without loss of generality we may consider that the equilibrium of $(2.2)$ is $x^{*}=0$.

Take $t \geq 0$ and $\varphi \in S$ with $\|\varphi\|_{\infty, d}=|\varphi(0)|_{\infty, d}>0$. Let $i$ be such $\|\varphi\|_{\infty, d}=d_{i}\left|\varphi_{i}(0)\right|$, and suppose that $\varphi_{i}(0)>0$ (the case $\varphi_{i}(0)<0$ is analogous). Then,

$$
b_{i}\left(\varphi_{i}(0)\right)+f_{i}(t, \varphi)=\left[b_{i}\left(\varphi_{i}(0)\right)-b_{i}(0)\right]+\left[f_{i}(t, \varphi)-f_{i}(t, 0)\right] \geq \beta_{i} \varphi_{i}(0)-l_{i}\|\varphi\|_{\infty, d}>0 .
$$

This proves that $(\mathrm{h})_{\infty}$ holds, and the result follows now from Proposition 2.1.

Proposition 2.4. Consider (2.2), write $f=\left(f_{1}, \ldots, f_{n}\right)$ and suppose that $\rho_{i}(t, \varphi)$ are uniformly positive and bounded, with

$$
0<r \leq \rho_{i}(t, \varphi) \leq R, \quad \text { for } \quad t \geq 0, \varphi \in S, i=1, \ldots, n,
$$

for some constants $r, R$. Assume (A1), (A2) and

$(A 3)_{2}$ there is a vector $d=\left(d_{1}, \ldots, d_{n}\right)>0$ such that $|f(t, \varphi)-f(t, \psi)|_{2, d} \leq l\|\varphi-\psi\|_{2, d}$, for all $t \geq 0$ and $\varphi, \psi \in S$, where the Lipschitz constant $l$ satisfies

$$
l \sqrt{R / r}<\min _{1 \leq i \leq n} \beta_{i} .
$$

Then, all solutions $x(t)=x\left(t, t_{0}, \varphi\right)$ (with $t_{0} \geq 0, \varphi \in S$ ) of (2.2) are defined and bounded on $\left[t_{0}, \infty\right)$ and satisfy $\left|x(t)-x^{*}\right|_{2, d} \leq\left\|\varphi-x^{*}\right\|_{2, d}$ for $t \geq t_{0}$.

Proof. As in the above proof, we may already assume that the equilibrium of $(2.2)$ is $x^{*}=0$. Write $g=\left(g_{1}, \ldots, g_{n}\right)$ with $g_{i}(t, \varphi)=\rho_{i}(t, \varphi)\left[b_{i}\left(\varphi_{i}(0)\right)+f_{i}(t, \varphi)\right]$ and let $\underline{\beta}=\min _{1 \leq i \leq n} \beta_{i}$.

Now, consider $t \geq 0$ and $\varphi \in S$ with $\|\varphi\|_{2, d}=|\varphi(0)|_{2, d}>0$. Define $c_{i}=d_{i} \rho_{i}(t, \varphi), c=$ $\left(c_{1}, \ldots, c_{n}\right)$, and note that (2.4) implies that $r^{1 / 2}|x|_{2, d} \leq|x|_{2, c} \leq R^{1 / 2}|x|_{2, d}$ for $x \in \mathbb{R}^{n}$. We get

$$
\begin{aligned}
<\varphi(0), g(t, \varphi)>_{d} & \geq \underline{\beta} \sum_{i=1}^{n} d_{i} \rho_{i}(t, \varphi) \varphi_{i}^{2}(0)-\sum_{i=1}^{n} d_{i} \rho_{i}(t, \varphi)\left|\varphi_{i}(0)\right|\left|f_{i}(t, \varphi)-f_{i}(t, 0)\right| \\
& =\underline{\beta}|\varphi(0)|_{2, c}^{2}-<|\varphi(0)|,|f(t, \varphi)-f(t, 0)|>_{c} \\
& \geq \underline{\beta}|\varphi(0)|_{2, c}^{2}-|\varphi(0)|_{2, c}|f(t, \varphi)-f(t, 0)|_{2, c},
\end{aligned}
$$


where we used the notation $|f|=\left(\left|f_{1}\right|, \ldots,\left|f_{n}\right|\right),|\varphi|=\left(\left|\varphi_{1}\right|, \ldots,\left|\varphi_{n}\right|\right)$. On the other hand, from (2.4) and (A3) $)_{2}$

$$
|f(t, \varphi)-f(t, 0)|_{2, c} \leq \sqrt{R}|f(t, \varphi)-f(t, 0)|_{2, d} \leq l \sqrt{R}|\varphi(0)|_{2, d} .
$$

From (2.5) and (2.6), we thus obtain

$$
<\varphi(0), g(t, \varphi)>_{d} \geq(\underline{\beta} \sqrt{r}-l \sqrt{R})|\varphi(0)|_{2, d}|\varphi(0)|_{2, c}>0 .
$$

The result is now a consequence of Proposition 2.2.

It is interesting to notice that for autonomous functions $f_{i}(t, \varphi) \equiv f_{i}(0, \varphi)$, or more generally when $f_{i}(t, \hat{x}) \equiv f_{i}(0, \hat{x})$ for $t \geq 0, x \in \mathbb{R}^{n}$, then (A2) and a Lipschitz condition on $f$ imply that there is a unique equilibrium $x^{*}$ of $(2.2)$, as shown below. This means that, in this case, (A1) simply requires that the equilibrium $x^{*}$ is in $S$.

Lemma 2.1. Consider (2.2), where $f_{i}(t, \hat{x}) \equiv f_{i}(0, \hat{x}):=f_{i}(x)$ for $t \geq 0, x \in \mathbb{R}^{n}$, with $f_{i}: \mathbb{R}^{n} \rightarrow$ $\mathbb{R}, i=1, \ldots, n$. Assume (A2), and either $(A 3)_{\infty}$ or $(A 3)_{2}$ with (2.5) replaced by $l<\min _{1 \leq i \leq n} \beta_{i}$. Then, there is a unique $x^{*}=\left(x_{i}^{*}, \ldots, x_{n}^{*}\right) \in \mathbb{R}^{n}$ such that $b_{i}\left(x_{i}^{*}\right)+f_{i}\left(x^{*}\right)=0, i=1, \ldots, n$.

Proof. If (A2) and (A3) $)_{\infty}$ hold, the result was shown in [17] (for related results, see also e.g. $[5,6,9,16])$, so here we only consider the situation for norms $|\cdot|_{2, d}$ in $\mathbb{R}^{n}$. Define $H: \mathbb{R}^{n} \rightarrow$ $\mathbb{R}^{n}, H(x)=b(x)+f(x)$, where

$$
b(x)=\left(b_{1}\left(x_{1}\right), \ldots, b_{n}\left(x_{n}\right)\right), f(x)=\left(f_{1}(x), \ldots, f_{n}(x)\right), \quad \text { for } \quad x=\left(x_{1}, \ldots, x_{n}\right) .
$$

If $x, y \in \mathbb{R}^{n}$ and $H(x)=H(y)$, then

$$
\begin{aligned}
\underline{\beta}^{2}|x-y|_{2, d}^{2} & \leq \sum_{i=1}^{n} d_{i} \beta_{i}^{2}\left|x_{i}-y_{i}\right|^{2} \leq \sum_{i=1}^{n} d_{i}\left|b_{i}\left(x_{i}\right)-b_{i}\left(y_{i}\right)\right|^{2} \\
& =\sum_{i=1}^{n} d_{i}\left|f_{i}(x)-f_{i}(y)\right|^{2} \leq l^{2}|x-y|_{2, d}^{2},
\end{aligned}
$$

with $l<\beta$. This shows that $H$ is injective. On the other hand, one writes $H(x)=(b(x)-b(0))+$ $(f(x)-f(0))+(b(0)+f(0))$, from which one deduces that

$$
|H(x)|_{2, d} \geq(\underline{\beta}-l)|x|_{2, d}-\mid\left(b(0)+\left.f(0)\right|_{2, d} \rightarrow \infty \quad \text { as } \quad|x|_{2, d} \rightarrow \infty .\right.
$$

By [9], this proves that $H$ is a homeomorfism of $\mathbb{R}^{n}$, hence there is a unique root $x^{*}$ of the equation $H(x)=0$.

\section{Exponential stability}

We now address the global exponential stability of $x^{*}$ by using the results in Section 2 . 
Theorem 3.1. Consider the FDE (2.2), with $S \subset C_{n}$ as the set of admissible initial conditions. Assume (A1), (A2), (A3) $\infty$ and

$$
\underline{\rho_{i}}:=\inf \left\{\rho_{i}(t, \varphi): t \geq t_{0}, \varphi \in S\right\}>0, \quad i=1, \ldots, n .
$$

Then, the equilibrium $x^{*}$ is globally exponentially stable (in the set of all admissible solutions).

Proof. As before, by translating the equilibrium to the origin we may assume that $x^{*}=0$, and thus $b_{i}(0)+f_{i}(t, 0)=0$ for $t \geq 0$ and $i=1, \ldots, n$. By Proposition 2.3, we know already that solutions satisfy $|x(t, 0, \varphi)|_{\infty, d} \leq\|\varphi\|_{\infty, d}$ for $t \geq 0$ and $\varphi \in S$.

Now, effect the change of variables $z(t)=e^{\varepsilon t} x(t)$ for $\varepsilon>0$. Eq. (2.2) translates as

$$
\dot{z}_{i}(t)=g_{i}\left(t, z_{t}\right), \quad t \geq 0, i=1, \ldots, n,
$$

where

$$
g_{i}(t, \varphi)=\varepsilon \varphi_{i}(0)-\rho_{i}\left(t, e^{-\varepsilon(t+\cdot)} \varphi\right) e^{\varepsilon t}\left[b_{i}\left(e^{-\varepsilon t} \varphi_{i}(0)\right)+f_{i}\left(t, e^{-\varepsilon(t+\cdot)} \varphi\right)\right] .
$$

Take $t \geq 0$ and $\varphi \in C_{n}$ with $e^{-\varepsilon(t+\cdot)} \varphi \in S$. Suppose that $\|\varphi\|_{\infty, d}=|\varphi(0)|_{\infty, d}>0$, and let $i$ be such $\|\varphi\|_{\infty, d}=d_{i}\left|\varphi_{i}(0)\right|_{\infty, d}$, with $\varphi_{i}(0)>0$ (the case $\varphi_{i}(0)<0$ is analogous). Then, for $\varepsilon$ such that $\beta_{i}-d_{i} l_{i} e^{\varepsilon \tau}>0$,

$$
\begin{aligned}
g_{i}(t, \varphi) & \leq \varepsilon \varphi_{i}(0)-\rho_{i}\left(t, e^{-\varepsilon(t+\cdot)} \varphi\right) e^{\varepsilon t} \varphi_{i}(0)\left[\beta_{i} e^{-\varepsilon t}-d_{i} l_{i} e^{-\varepsilon t} e^{\varepsilon \tau}\right] \\
& \leq \varphi_{i}(0)\left[\varepsilon-\underline{\rho_{i}}\left(\beta_{i}-d_{i} l_{i} e^{\varepsilon \tau}\right)\right] .
\end{aligned}
$$

Fix $\varepsilon>0$ such that $\varepsilon-\underline{\rho_{i}}\left(\beta_{i}-d_{i} l_{i} e^{\varepsilon \tau}\right)<0$. By applying Proposition 2.1, we deduce that the solutions $z(t)$ of (3.2) satisfy $|z(t)|_{\infty, d} \leq\left\|z_{0}\right\|_{\infty, d}$ for $t \geq 0$. Thus we obtain

$$
|x(t, 0, \varphi)|_{\infty, d}=\left|e^{-\varepsilon t} z\left(t, 0, e^{\varepsilon \cdot} \varphi\right)\right|_{\infty, d} \leq e^{-\varepsilon t}\|\varphi\|_{\infty, d}, \quad t \geq 0, \varphi \in S .
$$

Similarly, the application of Proposition 2.2 yields the following result:

Theorem 3.2. For (2.2) with $S \subset C_{n}$ as the set of admissible initial conditions, assume (2.4), (A1), (A2) and $(A 3)_{2}$. Then, the equilibrium $x^{*}$ is globally exponentially stable.

Proof. The proof follows along the lines above, so it is omitted.

As we shall see in Section 4, condition (3.1) in general fails for (2.2) describing a population dynamics model, hence Theorems 3.1 and 3.2 are not applicable.

\section{Applications}

In this section, we apply the stability criteria in Section 3 to some $n$-dimensional FDEs which have been widely used in both neural networks and population dynamics. 


\subsection{Neural Network models with delays}

As a particular class included in the framework set by (2.2), we consider generalizations of the Cohen-Grossberg neural network with distributed delays:

$$
\dot{x}_{i}(t)=-k_{i}\left(x_{i}(t)\right)\left[b_{i}\left(x_{i}(t)\right)+\sum_{j=1}^{n} f_{i j}\left(x_{j, t}\right)\right], \quad t \geq 0, \quad i=1, \ldots, n,
$$

where $k_{i}: \mathbb{R} \rightarrow(0,+\infty), b_{i}: \mathbb{R} \rightarrow \mathbb{R}$ e $f_{i j}: C_{1} \rightarrow \mathbb{R}$ are continuous, $i, j=1, \ldots, n$, and $x_{t}=\left(x_{1, t}, \ldots, x_{n, t}\right)$. Here, we take the entire phase space $C_{n}$ as the set of admissible initial conditions. We note that (4.1) generalizes several systems studied in [1, 2, 4-6, 17, 20-25]; see also references therein.

For (4.1), denote $f=\left(f_{1}, \ldots, f_{n}\right): C_{n} \rightarrow \mathbb{R}^{n}$, where

$$
f_{i}(\varphi)=\sum_{j=1}^{n} f_{i j}\left(\varphi_{j}\right), \quad \varphi=\left(\varphi_{1}, \ldots, \varphi_{n}\right) \in C_{n} .
$$

Proposition 4.1. For (4.1), assume that $b_{i}$ satisfy (A2), $f_{i j}$ are Lipschitzian with

$$
\left|f_{i j}(\varphi)-f_{i j}(\psi)\right| \leq l_{i j}\|\varphi-\psi\|_{\infty}, \quad \varphi, \psi \in C_{1}, i, j=1, \ldots, n,
$$

and

$$
\underline{k_{i}}:=\inf _{x \in \mathbb{R}} k_{i}(x)>0, \quad i=1, \ldots, n,
$$

For $\beta_{i}$ as in (A2), suppose in addition that the matrix

$$
N=\operatorname{diag}\left(\beta_{1}, \ldots, \beta_{n}\right)-\left[l_{i j}\right]
$$

is a non-singular M-matrix. Then, there is a unique equilibrium $x^{*}$ of (4.1), which is globally exponentially stable.

Proof. Eq. (4.1) has the form (2.2) with $f_{i}(t, \varphi)=f_{i}(\varphi)$ as in (4.2) and $\rho_{i}(t, \varphi)=k_{i}\left(\varphi_{i}(0)\right)$ for $t \geq 0, \varphi \in C_{n}$ and $i=1, \ldots, n$. If $N$ is a non-singular M-matrix, then there is $c=\left(c_{1}, \ldots, c_{n}\right)>0$ such that $N c>0[8$, Theorem 5.1], i.e.,

$$
\beta_{i}>c_{i}^{-1} \sum_{j=1}^{n} l_{i j} c_{j}, \quad i=1, \ldots, n .
$$

Clearly $(\mathrm{A} 3)_{\infty}$ holds with $d=\left(c_{1}^{-1}, \ldots, c_{n}^{-1}\right)$. The result is a consequence of Lemma 2.1 and Theorem 3.1.

We remark that system (4.1) is sufficiently general to include most of the autonomous delayed neural network models considered in the literature, namely Hopfield, Cohen-Grossberg, static, bidirectional associative memory models. Proposition 4.1 strongly improves several criteria established in e.g. [6, Theorem 2], [16, Theorems 2.1 and 2.2], [22, Theorem 3.1].

In an analogous way, we now apply Theorem 3.2 to (4.1) (for comparison of results, see e.g. $[2,3,15,21,22])$. 
Proposition 4.2. For (4.1), assume (A2) and that there are constants $\underline{k}, \bar{k}>0$ such that

$$
\underline{k} \leq k_{i}(x) \leq \bar{k}, \quad \text { for } \quad x \in \mathbb{R}, i=1, \ldots, n .
$$

Suppose in addition that there is $d=\left(d_{1}, \ldots, d_{n}\right)>0$ such that

$$
|f(\varphi)-f(\psi)|_{2, d} \leq l\|\varphi-\psi\|_{2, d}, \quad \varphi, \psi \in C_{n}
$$

with

$$
l \sqrt{\bar{k} / \underline{k}}<\min _{1 \leq i \leq n} \beta_{i}
$$

for $\beta_{i}$ as in (A2). Then, there is an equilibrium $x^{*}$ of (4.1), which is globally exponentially stable.

Recently, the following Cohen-Grossberg model with time-dependent discrete delays has been studied $[3,12,25]$ :

$$
\dot{x}_{i}(t)=-k_{i}\left(x_{i}(t)\right)\left[b_{i}\left(x_{i}(t)\right)+\sum_{j=1}^{n} \sum_{p=1}^{P} h_{i j p}\left(x_{j}\left(t-\tau_{i j p}(t)\right)\right)+J_{i}\right], \quad i=1, \cdots, n, t \geq 0,
$$

where: $k_{i}: \mathbb{R} \rightarrow(0,+\infty), b_{i}, h_{i j p}: \mathbb{R} \rightarrow \mathbb{R}, \tau_{i j p}:[0,+\infty) \rightarrow[0,+\infty)$ are continuous functions, with $k_{i}$ uniformly positive on $[0,+\infty), J_{i} \in \mathbb{R}$ and $\tau_{i j p}$ bounded, $i, j=1, \ldots, n, p=1, \ldots, P$.

For $\tau \geq 0$ such that $\tau_{i j p}(t) \in[0, \tau]$ for $t \geq 0$ and $i, j=1, \ldots, n, p=1, \ldots, P$, we take $C_{n}=C\left([-\tau, 0] ; \mathbb{R}^{n}\right)$ as the phase space for (4.9) as well as the set of admissible initial conditions.

Proposition 4.3. For system (4.9), assume (A2), (4.4) and that $h_{i j p}$ are Lipschitz continuous with Lipschitz constants $l_{i j p}$ for $i, j=1, \ldots, n, p=1, \ldots, P$. For $\beta_{i}$ as in (A2) and $l_{i j}=\sum_{p=1}^{P} l_{i j p}$, suppose also that the matrix $N=\operatorname{diag}\left(\beta_{1}, \ldots, \beta_{n}\right)-\left[l_{i j}\right]$ is a non-singular $M$-matrix. Then, there is a unique equilibrium $x^{*}$ of (4.9), which is globally exponentially stable.

Proof. Write (4.9) as (2.2), where

$$
f_{i}(t, \varphi)=\sum_{j=1}^{n} \sum_{p=1}^{P} h_{i j p}\left(\varphi_{j}\left(-\tau_{i j p}(t)\right)\right)+J_{i}, \quad \rho_{i}(t, \varphi)=k_{i}\left(\varphi_{i}(0)\right), \quad i=1, \ldots, n,
$$

for $t \geq 0, \varphi \in C_{n}$. Let $c=\left(c_{1}, \cdots, c_{n}\right)>0$ be such that (4.5) holds. For $\varphi, \psi \in C_{n}$ and $t \geq 0$ we have

$$
\left|f_{i}(t, \varphi)-f_{i}(t, \psi)\right| \leq \sum_{j=1}^{n} \sum_{p=1}^{P} c_{j} l_{i j p}\|\varphi-\psi\|_{\infty, c^{-1}}=\left(\sum_{j=1}^{n} c_{j} l_{i j}\right)\|\varphi-\psi\|_{\infty, c^{-1}},
$$

and therefore assumption $(\mathrm{A} 3)_{\infty}$ holds with $d=c^{-1}:=\left(c_{1}^{-1}, \cdots, c_{n}^{-1}\right)>0$ and $l_{i}=\sum_{j=1}^{n} c_{j} l_{i j}$. Again, the result follows from Lemma 2.1 and Theorem 3.1.

The exponential stability for Eq. (4.9) was investigated in [12]. We note however that our Proposition 4.3 significantly improves the following criterion given in [12, Theorem 4]: 
Proposition 4.4. [12] Consider Eq. (4.9) with $h_{i j p}(x)=c_{i j p} g_{i j p}(x)$, for $c_{i j p} \in \mathbb{R}$ and $g_{i j p}(x)$ continuous functions. Assume that, for $i, j=1, \ldots, n$ and $p=1, \ldots, P$ :

(i) $k_{i}(x)$ are locally Lipschitz continuous and there are constants $\overline{k_{i}}, \underline{k_{i}}>0$ such that

$$
\underline{k_{i}} \leq k_{i}(x) \leq \overline{k_{i}}, \quad x \in \mathbb{R}
$$

(ii) $b_{i} \in C^{1}(\mathbb{R}, \mathbb{R})$ with $b_{i}^{\prime}(x) \geq \beta_{i}>0$ for $x \in \mathbb{R}$;

(iii) $g_{i j p}$ are bounded Lipschitzian functions, with Lipschitz constants $\mu_{i j p}$;

(iv) for $\overline{k_{i}}, \underline{k_{i}}$ as in (i) and $\beta_{i}$ as in (ii), there are constants $\alpha_{i j p}, \gamma_{i j p} \in \mathbb{R}, \omega_{i}>0, r>1$ and $\sigma>0$ such that

$$
r \omega_{i} \underline{k_{i}} \beta_{i}-(r-1) \sum_{j=1}^{n} \sum_{p=1}^{P} \omega_{j} \overline{k_{i}} \mu_{i j p}^{\frac{r-\gamma_{i j p}}{r-1}}\left|c_{i j p}\right|^{\frac{r-\alpha_{i j p}}{r-1}}-\sum_{j=1}^{n} \sum_{p=1}^{P} \omega_{j} \overline{k_{i}} \mu_{i j p}^{\gamma_{i j p}}\left|c_{i j p}\right|^{\alpha_{i j p}}>\sigma .
$$

Then, there is a unique equilibrium $x^{*}$ of (4.9), which is globally exponentially stable.

Comparing the hypotheses in Proposition 4.3 and Proposition 4.4, we remark that, in the latter case, not only the constraints imposed on the functions $k_{i}, b_{i}$ and $g_{i j p}$ are clearly more restrictive, but also condition (4.10) is stronger than the requirement of $N$ being a non-singular M-matrix, as shown in the next Lemma. See also Example 5.3.

Lemma 4.1. With the above notation, suppose that there are constants $r>1, \sigma>0, \alpha_{i j p}, \gamma_{i j p} \in$ $\mathbb{R}, \omega_{i}>0$, for $i, j=1, \ldots, n$ and $p=1, \ldots, P$, for which condition (4.10) is satisfied. Then, for $l_{i j}=\sum_{p=1}^{P}\left|c_{i j p}\right| \mu_{i j p}$, the matrix $N=\operatorname{diag}\left(\beta_{1}, \ldots, \beta_{n}\right)-\left[l_{i j}\right]$ is a non-singular M-matrix.

Proof. Condition (4.10) can be written as

$$
r \omega_{i} \underline{k_{i}} \beta_{i}-\overline{k_{i}} \sum_{j=1}^{n} \omega_{j} \sum_{p=1}^{P}\left((r-1) \mu_{i j p}^{\frac{r-\gamma_{i j p}}{r-1}}\left|c_{i j p}\right|^{\frac{r-\alpha_{i j p}}{r-1}}+\mu_{i j p}^{\gamma_{i j p}}\left|c_{i j p}\right|^{\alpha_{i j p}}\right)>\sigma,
$$

or, equivalently,

$$
\omega_{i} \underline{k_{i}} \beta_{i}>\frac{\sigma}{r}+\overline{k_{i}} \sum_{j=1}^{n} \omega_{j} \sum_{p=1}^{P}\left[\frac{r-1}{r}\left(\mu_{i j p}^{1-\frac{\gamma_{i j p}}{r}}\left|c_{i j p}\right|^{1-\frac{\alpha_{i j p}}{r}}\right)^{\frac{r}{r-1}}+\frac{1}{r}\left(\mu_{i j p}^{\frac{\gamma_{i j p}}{r}}\left|c_{i j p}\right|^{\frac{\alpha_{i j p}}{r}}\right)^{r}\right] .
$$

Applying Young's inequality (see e.g. [12]), we therefore obtain

$$
\omega_{i} \underline{k_{i}} \beta_{i}>\frac{\sigma}{r}+\overline{k_{i}} \sum_{j=1}^{n} \omega_{j} \sum_{p=1}^{P} \mu_{i j p}\left|c_{i j p}\right| \text {. }
$$

Since $\overline{k_{i}} \geq \underline{k_{i}}>0$, from (4.11) we have $\omega_{i} \beta_{i}>\sum_{j=1}^{n} \omega_{j} l_{i j}, i=1, \ldots, n$, from which we conclude that $N$ is a non-singular M-matrix [8]. 


\subsection{Delayed Kolmogorov-type population models}

Consider multiple species Kolmogorov systems with delays and instantaneous negative feedbacks given by

$$
\dot{x}_{i}(t)=r_{i}(t) x_{i}(t)\left[-b_{i} x_{i}(t)+f_{i}\left(x_{t}\right)\right] \quad t \geq 0, \quad i=1, \ldots, n,
$$

where $b_{i}>0, r_{i}:[0, \infty) \rightarrow(0, \infty)$ are continuous and $f_{i}: C_{n} \rightarrow \mathbb{R}$ are Lipschitz continuous functions, $1 \leq i \leq n$. In a more general way, we can also study models with non-autonomous competition interactions $f_{i}\left(t, x_{t}\right)$, instead of $f_{i}\left(x_{t}\right)$, and suppose that $f_{i}(t, \varphi)$ are continuous and uniform Lipschitzian with respect to $\varphi$. For biological discussions and related results, see $[7,11$, $13,14]$, also for cited references.

In biological terms, only positive solutions of (4.12) are meaningful. We take

$$
C_{\hat{0}}=\left\{\varphi=\left(\varphi_{1}, \ldots, \varphi_{n}\right) \in C_{n}: \varphi_{i}(\theta) \geq 0 \text { for } \theta \in[-\tau, 0), \varphi_{i}(0)>0, i=1, \ldots, n\right\},
$$

as the set of admissible initial conditions, and only consider solutions of (4.12) with initial conditions $x_{t_{0}}=\varphi \in C_{\hat{0}}$ for some $t_{0} \geq 0$. A solution $x\left(t, t_{0}, \varphi\right)$ with initial condition in $C_{\hat{0}}$ is admissible, since $x_{i}\left(t, t_{0}, \varphi\right)=x_{i}\left(t_{0}\right) \exp \left(\int_{t_{0}}^{t} r_{i}(s)\left[-b_{i} x_{i}(s)+f_{i}\left(x_{s}\right)\right] d s\right)>0$, for $t>t_{0}$ whenever it is defined.

If $x^{*}=\left(x_{1}^{*}, \ldots, x_{n}^{*}\right)$ is a positive equilibrium of $(4.1)$, for $y_{i}(t)=x_{i}(t)-x_{i}^{*} \operatorname{system}(4.12)$ becomes

$$
\dot{y}_{i}(t)=-r_{i}(t)\left(y_{i}(t)+x_{i}^{*}\right)\left[b_{i} y_{i}(t)-f_{i}\left(y_{t}+x^{*}\right)+f_{i}\left(x^{*}\right)\right], i=1, \ldots, n,
$$

with $C_{\hat{0}}-x^{*}:=C_{-x^{*}}$ as the set of admissible initial conditions.

Proposition 4.5. For (4.12), suppose that there is a positive equilibrium $x^{*}=\left(x_{1}^{*}, \ldots, x_{n}^{*}\right)$, and that there is $d=\left(d_{1}, \ldots, d_{n}\right)>0$ such that for $\varphi, \psi \in C_{\hat{0}}$ and $i=1, \ldots, n$,

$$
\left|f_{i}(\varphi)-f_{i}(\psi)\right| \leq l_{i}\|\varphi-\psi\|_{\infty, d}, \quad \text { with } \quad d_{i} l_{i}<b_{i}
$$

(i) If

$$
\underline{r_{i}}:=\inf _{t \geq 0} r_{i}(t)>0, i=1, \ldots, n,
$$

then, for any $\delta>0$ small, $x^{*}$ is globally exponentially stable in the set of solutions satisfying $x_{i}(t) \geq \delta$ for $t \geq 0, i=1, \ldots, n$.

(ii) If (4.14) also holds with $\varphi, \psi \in \overline{C_{\hat{0}}}$ and

$$
\sup _{t \geq 0} r_{i}(t):=\overline{r_{i}}<\infty \quad \text { and } \quad \int^{\infty} r_{i}(t) d t=\infty, \quad i=1, \ldots, n
$$

then $x^{*}$ is globally asymptotically stable.

Proof. After translating $x^{*}$ to the origin, consider (4.13). This equation has the form (2.2), for which condition $(\mathrm{A} 3)_{\infty}$ is satisfied by virtue of (4.14). 
For the proof of (i), we now assume (4.15), and fix $\delta>0$ with $\delta<x_{i}^{*}, i=1, \ldots, n$. Consider (4.13) with $S_{\delta}=\left\{\left(\varphi_{1}, \ldots, \varphi_{n}\right) \in C_{n}: \varphi_{i}(\theta)+x_{i}^{*} \geq \delta\right.$ for $\left.\tau \leq \theta \leq 0, i=1, \ldots, n\right\}$ as the set of admissible initial conditions. We have $\rho_{i}(t, \varphi):=r_{i}(t)\left(\varphi_{i}(0)+x_{i}^{*}\right) \geq \delta \underline{r_{i}}$ for $t \geq 0, \varphi \in S_{\delta}, i=$ $1, \ldots, n$. Thus, Theorem 3.1 implies the global exponential stability of zero on $S_{\delta}$.

Now, suppose that (4.16) holds. From Proposition 2.3, all solutions of (4.13) are defined and bounded on $[0, \infty)$ and the origin is uniformly stable. It remains to prove that $y(t) \rightarrow 0$ as $t \rightarrow \infty$, for all (admissible) solutions of (4.13). We now argue as in the proof of [7, Theorem 3.4], where details can be found.

After the scaling $y_{i}(t) \mapsto d_{i} y_{i}(t), i=1, \ldots, n$, we may assume that (4.14) holds with $d=$ $(1, \ldots, 1)$. Let $y(t)=\left(y_{i}(t)\right)_{i=1}^{n}$ be a solution to (4.13), and set

$$
\begin{gathered}
-v_{i}=\liminf _{t \rightarrow \infty} y_{i}(t), \quad u_{i}=\limsup _{t \rightarrow \infty} y_{i}(t), \quad i=1, \ldots, n, \\
v=\max _{1 \leq i \leq n} v_{i}, \quad u=\max _{1 \leq i \leq n} u_{i} .
\end{gathered}
$$

Note that $-x_{i}^{*} \leq-v_{i} \leq u_{i}<\infty, i=1, \ldots, n$. It is sufficient to prove that $\max (u, v)=0$. Assume e.g. that $v \leq u$, so that $\max (u, v)=u$ (the case $u \leq v$ is analogous). Let $i$ be such that $u_{i}=u$, and fix $\varepsilon>0$ small. Proceeding as in [7], we can show that there is a sequence $t_{k} \rightarrow \infty$ such that

$$
y_{i}\left(t_{k}\right) \rightarrow u, \quad b_{i} y_{i}\left(t_{k}\right)-f_{i}\left(y_{t_{k}}+x^{*}\right)+f_{i}\left(x^{*}\right) \rightarrow 0 \quad \text { and } \quad\left\|y_{t_{k}}\right\|_{\infty} \leq u+\varepsilon
$$

The boundedness of $y(t)$ and $r_{i}(t)$ implies that $\dot{y}(t)$ is also bounded on $[0, \infty)$. Hence, the sequence $\left(y_{t_{k}}\right)$ in $C_{n}$ is uniformly bounded and equicontinuous. Up to a subsequence, we may suppose that $y_{t_{k}} \rightarrow \varphi$ in $C_{n}$, for some $\varphi \in \overline{C_{-x^{*}}}$. From (4.17), by letting $k \rightarrow \infty$ and $\varepsilon \rightarrow 0$ we obtain

$$
\varphi_{i}(0)=u, \quad b_{i} u-f_{i}\left(\varphi+x^{*}\right)+f_{i}\left(x^{*}\right)=0 \quad \text { and } \quad\|\varphi\|_{\infty}=u .
$$

From (4.14) and (4.18), we deduce that $0 \geq b_{i} u-l_{i}\|\varphi\|_{\infty}=\left(b_{i}-l_{i}\right) u$. Since $l_{i}<b_{i}$ this implies that $u=0$, showing that $y(t) \rightarrow 0$ as $t \rightarrow \infty$. The proof is complete.

If $f_{i}(\varphi)=\sum_{i=1}^{n} f_{i j}\left(\varphi_{j}\right)$ for $\varphi=\left(\varphi_{1}, \ldots, \varphi_{n}\right)$ where $f_{i j}: C_{1} \rightarrow \mathbb{R}$ satisfy the Lipschitz conditions in (4.3), then, as proven in Section 4.1, there is a positive vector $d$ for which (4.14) is satisfied if and only if the matrix $N=\operatorname{diag}\left(b_{1}, \ldots, b_{n}\right)-\left[l_{i j}\right]$ is a non-singular M-matrix.

Remark 4.1. As a particular case of (4.12), consider Lotka-Volterra type systems

$$
\dot{x}_{i}(t)=r_{i}(t) x_{i}(t)\left[\alpha_{i}-b_{i} x_{i}(t)-\sum_{j=1}^{n} l_{i j} \int_{-\tau}^{0} x_{j}(t+\theta) d \eta_{i j}(\theta)\right], \quad i=1, \ldots, n,
$$

where $\alpha_{i} \in \mathbb{R}, b_{i}>0, l_{i j} \geq 0, \tau>0, r_{i}(t)$ are positive continuous functions and $\eta_{i j}:[-\tau, 0] \rightarrow \mathbb{R}$ are normalized bounded variation functions. If $N=\operatorname{diag}\left(b_{1}, \ldots, b_{n}\right)-\left[l_{i j}\right]$ is a non-singular M-matrix and (4.16) holds, from Proposition 4.5 then the positive equilibrium $x^{*}$ (if it exists) is globally 
asymptotically stable. In [7], this stability was proven by imposing (4.16), that $N$ is an M-matrix, and replacing $\operatorname{det} N \neq 0$ by the weaker condition $\operatorname{det} \tilde{M} \neq 0$, for $\tilde{M}=\operatorname{diag}\left(b_{1}, \ldots, d_{n}\right)+\left[\tilde{a}_{i j}\right]$, where $\tilde{a}_{i i}=a_{i i}, \tilde{a}_{i j}=-\left|a_{i j}\right|$ for $i \neq j$, and $a_{i j}=l_{i j} \int_{-\tau}^{0} d \eta_{i j}(\theta)$. For more results, see also [11].

Remark 4.2. From Lemma 2.1, condition (4.14) implies that there is an equilibrium $x^{*}$ of (4.12), but possibly non-positive. Note also that, for population models (4.12), the stability result in Proposition 4.5 is weaker than the criterion in Proposition 4.1 for neural networks, due to the fact that $\inf _{\varphi \in C_{-x^{*}}}\left(\varphi_{i}(0)+x_{i}^{*}\right)=0$, so that Theorem 3.1 cannot be directly applied. On the other hand, our boundedness and stability results relative to norms $|\cdot|_{2, d}$ are not of interest in the present situation, since their application requires the estimates (2.4) and (2.5). We should however mention that Saito et al. [18] and Saito and Takeuchi [19] considered a predator-prey model (4.19) with $n=2$ and the symmetry given by $N=\operatorname{diag}(b, b)-L$, for $L:=\left(\begin{array}{cc}\alpha & \beta \\ -\beta & \alpha\end{array}\right)$ and $N$ defined as above, and showed its global asymptotic stability if and only if $\sqrt{\alpha^{2}+\beta^{2}} \leq b$. Note that in this case we can choose $l=\|L\|_{2}=\sqrt{\alpha^{2}+\beta^{2}}$ for the Lipschitz constant $l$ in (4.7).

\section{Examples}

We complement the above criteria with some illustrative examples.

Example 5.1. Consider the two neuron network with distributed delays

$$
\begin{aligned}
& \dot{x}_{1}(t)=-b_{1} x_{1}(t)+a_{11} f_{11}\left(x_{1, t}\right)+a_{12} f_{12}\left(x_{2, t}\right) \\
& \dot{x}_{2}(t)=-b_{2} x_{2}(t)+a_{21} f_{21}\left(x_{1, t}\right)+a_{22} f_{22}\left(x_{2, t}\right)
\end{aligned}
$$

where $b_{i}>0, a_{i j} \in \mathbb{R}$ and $f_{i j}: C_{1}:=C([-\tau, 0] ; \mathbb{R}) \rightarrow \mathbb{R}$ are Lipschitzian functions normalized so that $\left|f_{i j}(\varphi)-f_{i j}(\psi)\right| \leq\|\varphi-\psi\|_{\infty}$ for $\varphi, \psi \in C_{1}, i, j=1,2$.

Define $N=\operatorname{diag}\left(b_{1}, b_{2}\right)-\left[\left|a_{i j}\right|\right]$. By [8, Theorem 5.1], $N$ is a non-singular M-matrix if and only if all its principal minors are positive, which translates here as

$$
b_{1}>\left|a_{11}\right| \text { and }\left(b_{1}-\left|a_{11}\right|\right)\left(b_{2}-\left|a_{22}\right|\right)>\left|a_{12} a_{21}\right| .
$$

By Proposition 4.1 and Lemma 2.1, (5.2) implies that there is a globally exponentially stable equilibrium $x^{*}$. That is the case if, for instance, $b_{1}=b_{2}=2.1, a_{11}=a_{22}=1, a_{12}=1 / 2, a_{21}=2$.

With $b_{i}, f_{i j}$ as in (5.1), consider now the 2 -species delayed population system

$$
\begin{aligned}
& \dot{x}_{1}(t)=x_{1}(t)\left[\alpha_{1}-b_{1} x_{1}(t)-a_{11} f_{11}\left(x_{1, t}\right)-a_{12} f_{12}\left(x_{2, t}\right)\right] \\
& \dot{x}_{2}(t)=x_{2}(t)\left[\alpha_{2}-b_{2} x_{2}(t)-a_{21} f_{21}\left(x_{1, t}\right)-a_{22} f_{22}\left(x_{2, t}\right)\right] .
\end{aligned}
$$

Assuming (5.2) and the existence of a positive equibrium $x^{*}$, from Proposition 4.5 we conclude that $x^{*}$ is globally asymptotically stable and that, for each $\delta>0$ with $\delta<x_{1}^{*}, x_{2}^{*}, x^{*}$ is globally exponentially stable in the set of solutions $x(t)$ such that $x_{i}(t) \geq \delta, i=1,2, t \geq 0$. 
Example 5.2. Consider the 2-neuron network with one discrete delay

$$
\begin{aligned}
& \dot{x}_{1}(t)=-b_{1} x_{1}(t)+a_{11} f_{1}\left(x_{1}(t-\tau)\right)+a_{12} f_{2}\left(x_{2}(t-\tau)\right) \\
& \dot{x}_{2}(t)=-b_{2} x_{2}(t)+a_{21} f_{1}\left(x_{1}(t-\tau)\right)+a_{22} f_{2}\left(x_{2}(t-\tau)\right) .
\end{aligned}
$$

Here, $\tau, b_{i}, a_{i j}$ are constants, $\tau, b_{1}, b_{2}>0, f_{i}: \mathbb{R} \rightarrow \mathbb{R}$ are Lipschitz continuous functions, normalized so that $\left|f_{i}(u)-f_{i}(v)\right| \leq|u-v|, u, v \in \mathbb{R}$, for $i, j=1,2$. The coefficients of the connection matrix $A:=\left[a_{i j}\right]$ are arbitrary real numbers, representing either excitatory or inhibitory connections, according to their signs. We assume that there are self-connections, i.e., $a_{i i} \neq 0$. Without loss of generality we take $a_{11}, a_{22}>0$.

We first suppose that $a_{12} a_{21}<0$. Define

$$
f(\varphi)=A\left(\begin{array}{c}
f_{1}\left(\varphi_{1}(-\tau)\right) \\
f_{2}\left(\varphi_{2}(-\tau)\right)
\end{array}\right), \quad \varphi=\left(\varphi_{1}, \varphi_{2}\right) \in C_{2}
$$

Choose $d=\left(d_{1}, d_{2}\right)>0$ such that $d_{1} a_{11} a_{12}+d_{2} a_{21} a_{22}=0$. Then for $\varphi, \psi \in C_{2}$ we have

$$
\begin{aligned}
|f(\varphi)-f(\psi)|_{2, d}= & {\left[\left(d_{1} a_{11}^{2}+d_{2} a_{21}^{2}\right)\left(f_{1}\left(\varphi_{1}(-\tau)\right)-f_{1}\left(\psi_{1}(-\tau)\right)\right)^{2}\right.} \\
& \left.+\left(d_{1} a_{12}^{2}+d_{2} a_{22}^{2}\right)\left(f_{2}\left(\varphi_{2}(-\tau)\right)-f_{2}\left(\psi_{2}(-\tau)\right)\right)^{2}\right]^{1 / 2} \\
= & (\operatorname{det} A)^{1 / 2}\left[d_{1} \frac{a_{11}}{a_{22}}\left(f_{1}\left(\varphi_{1}(-\tau)\right)-f_{1}\left(\psi_{1}(-\tau)\right)\right)^{2}\right. \\
& \left.+d_{2} \frac{a_{22}}{a_{11}}\left(f_{2}\left(\varphi_{2}(-\tau)\right)-f_{2}\left(\psi_{2}(-\tau)\right)\right)^{2}\right]^{1 / 2} \\
\leq & \left(\max \left\{\frac{a_{11}}{a_{22}}, \frac{a_{22}}{a_{11}}\right\} \operatorname{det} A\right)^{1 / 2}\|\varphi-\psi\|_{2, d} .
\end{aligned}
$$

Hence, for $d>0$ chosen as above, $f$ given by (5.4) satisfies (4.8) if

$$
\left(\max \left\{\frac{a_{11}}{a_{22}}, \frac{a_{22}}{a_{11}}\right\} \operatorname{det} A\right)^{1 / 2}<\min \left\{b_{1}, b_{2}\right\} .
$$

In this situation, Lemma 2.1 and Proposition 4.2 imply that there is an equilibrium point $x^{*}$ of (5.3) which is globally exponentially stable.

Now let $a_{11}, a_{22}>0$ as before, but consider both situations $a_{12} a_{21}<0$ and $a_{12} a_{21}>0$. Define $N=\operatorname{diag}\left(b_{1}, b_{2}\right)-\left[\left|a_{i j}\right|\right]$. As in example $5.1, N$ is a non-singular M-matrix if

$$
b_{1}>a_{11} \text { and }\left(b_{1}-a_{11}\right)\left(b_{2}-a_{22}\right)>\left|a_{12} a_{21}\right|,
$$

in which case Proposition 4.1 allows to conclude the global exponential stability of $x^{*}$.

For instance, take system (5.3), with $b_{1}=b_{2}=2.1, a_{11}=a_{22}=1$ and $a_{12} a_{21}=-3$. In this case, (5.6) fails but (5.5) holds. Reversely, if $b_{1}=1.5, b_{2}=7.1, a_{11}=a_{22}=1$ and $a_{12} a_{21}=-3$, 
then (5.5) fails but (5.6) is satisfied. In both situations, we conclude that the equilibrium $x^{*}$, which exists by Lemma 2.1, is a global attractor of all solutions of (5.3), with an exponential rate of attraction. This shows the advantage of the possible use of both Euclidean and maximum norms (with weights $d_{i}$ ) in $\mathbb{R}^{n}$ accounted for by our method.

Example 5.3. Consider the neural network model

$$
\begin{aligned}
\dot{x}_{1}(t)=-\left(8+2 \sin x_{1}(t)\right) & {\left[x_{1}(t)-\tanh x_{1}(t)-2 \tanh x_{2}(t)-\tanh \left(x_{1}\left(t-\frac{1}{3} \sin t-1\right)\right)\right.} \\
& \left.-\tanh \left(x_{2}\left(t-\frac{1}{4} e^{-\sin t}-1\right)\right)+2\right] \\
\dot{x}_{2}(t)=-\left(5+2 \cos x_{2}(t)\right) & {\left[10 x_{2}(t)-2 \tanh x_{1}(t)-\tanh x_{2}(t)-\tanh \left(x_{1}\left(t-\frac{1}{4} e^{-\sin t}-1\right)\right)\right.} \\
& \left.-2 \tanh \left(x_{2}\left(t-\frac{1}{3} \sin t-1\right)\right)+3\right] .
\end{aligned}
$$

System (5.7) is very similar to the one considered in [12, Example 2]. It has the form (4.9), and satisfies all the assumptions in Proposition 4.3 , with $\underline{k_{1}}=6, \underline{k_{2}}=3, \beta_{1}=7, \beta_{2}=10$ and $N=\operatorname{diag}(7,10)-\left(\begin{array}{ll}2 & 3 \\ 3 & 3\end{array}\right)$, clearly a non-singular M-matrix. From Proposition 4.3. the unique equilibrium of (5.7) is globally exponentially stable.

We now show that (5.7) does not satisfy condition (4.10), so that the criterion of Jiang et al. [12] given in Proposition 4.4 cannot be invoked. Besides the above coefficients, we have $\overline{k_{1}}=$ $10, \overline{k_{2}}=7, \mu_{i j p}=1, i, j, p=1,2, c_{111}=c_{112}=c_{121}=c_{221}=c_{212}=-1, c_{121}=c_{211}=c_{222}=-2$, thus conditions (i)-(iii) in the statement of Proposition 4.4 are satisfied; for the sake of contraction, suppose that condition (iv) there also holds. From (4.10), we derive (4.11), translated here as

$$
\left\{\begin{array}{r}
22 \omega_{1}-30 \omega_{2}>\sigma / r \\
-21 \omega_{1}+9 \omega_{2}>\sigma / r
\end{array}\right.
$$

for some constants $\omega_{1}, \omega_{2}, \sigma>0$ and $r>1$. Since $A=\left(\begin{array}{cc}22 & -30 \\ -21 & 9\end{array}\right)$ is not a non-singular Mmatrix, then it is not possible to find a positive vector $\omega=\left(\omega_{1}, \omega_{2}\right)$ such that $A \omega>0$, which contradicts (5.8). This shows the advantage of the stability criterion given by Proposition 4.3 over the result established in [12].

\section{References}

[1] C. Bai, Global exponential stability and existence of periodic solution of Cohen-Grossberg type neural networks with delays and impulses, Nonlinear Anal. Real World Appl. 9 (2008) $747-761$.

[2] J. Cao, New results concerning exponential stability and periodic solutions of delayed cellular neural networks, Phys. Lett. A, 307 (2003) 136-147. 
[3] J. Cao and J. Liang, Boundedness and stability for Cohen-Grossberg neural network with time-varying delays, J. Math. Anal. Appl. 296 (2004) 665-685.

[4] J. Cao and Q. Song, Stability in Cohen-Grossberg-type bidirectional associative memory neural networks with time-varying delays, Nonlinearity 19 (2006) 1601-1617.

[5] J. Cao and J. Wang, Global exponential stability and periodicity of recurrent neural networks with time delays, IEEE Trans. Circuits Syst. 52 (2005) 920-931.

[6] Y. Chen, Global asymptotic stability of a general class of recurrent neural networks with time-varying delays, IEEE Trans. Circuits Syst. 52 (2005) 417-426.

[7] T. Faria and J. Oliveira, Local and global stability for Lotka-Volterra systems with distributed delays and instantaneous feedbacks, J. Differential Equations 244 (2008) 1049-1079.

[8] M. Fiedler, Special Matrices and Their Applications in Numerical Mathematics, Martinus Nijhoff Publ. (Kluwer), Dordrecht, 1986.

[9] M. Forti and A. Tesi, New conditions for global stability of neural networks with applications to linear and quadratic programming problems, IEEE Trans.Circuits Syst.I 42 (1995) 354-366.

[10] K. Gopalsamy, Stability of artificial neural networks with impulses, Appl. Math. Comput. 4 (2004) 783-813.

[11] J. Hofbauer and J. So, Diagonal dominance and harmless off-diagonal delays, Proc. Amer. Math. Soc. 128 (2000) 2675-2682.

[12] H. Jiang, J. Cao and Z. Teng, Dynamics of Cohen-Grossberg neural networks with time-varying delays, Phys. Lett. A 354 (2006) 414-422.

[13] Y. Kuang, Global stability in delayed nonautonomous Lotka-Volterra type systems without saturated equilibrium, Differential and Integral Equations 9 (1996) 557-567.

[14] Y. Kuang and H. L. Smith, Global stability for infinite delay Lotka-Volterra type systems, J. Differential Equations 103 (1993) 221-246.

[15] X. Liao, C. Li and K. Wong, Criteria for exponential stability of Cohen-Grossberg neural networks, Neural Networks 17 (2004) 1401-1414.

[16] S. Mohamad and K. Gopalsamy, Exponential stability of continuous-time and discrete-time cellular neural networks with delays, Appl. Math. Comput. 135 (2003) 17-38.

[17] J. Oliveira, Global asymptotic stability for neural networks models, preprint.

[18] Y. Saito, T. Hara and W. Ma, Necessary and sufficient conditions for permanence and global stability of a Lotka-Volterra system with two delays, J. Math. Anal. Appl. 236 (1999) 534-556.

[19] Y. Saito and Y. Takeuchi, Sharp conditions for global stability of Lotka-Volterra systems with delayed intraspecific competitions, Fields Inst. Commun. 36 (2003) 195-211.

[20] Q. Song and J. Cao, Global exponential stability of bidirectional associative memory neural networks with distributed delays, J. Comput. Appl. Math. 202 (2007) 266-279.

[21] P. van den Driessche and X. Zou, Global stability in delayed Hopfield neural network models, 
SIAM J. Appl. Math. 58 (1998) 1878-1890.

[22] L. Wang and X. Zou, Harmless delays in Cohen-Grossberg neural networks, Physica D 170 (2002) 162-173.

[23] L. Wang and X. Zou, Stability and bifurcation of bidirectional associative memory neural networks with delayed self-feedback, Internat. J. Bifur. Chaos Appl. Sci. Engrg. 15 (2005), $2145-2159$.

[24] X. Yang, C. Li, X. Liao, D. Evans and G. Megson, Global exponential periodicity of a class of bidirectional associative memory networks with finite distributed delays, Appl. Math. Comput. 171 (2005) 108-121.

[25] Z. Yang and D. Xu, Implulsive effects on stability of Cohen-Grossberg neural networks with variable delays, Appl. Math. Comput. 177 (2006) 63-78. 